\title{
How should eosinophilic cystitis be treated in patients with chronic granulomatous disease?
}

\author{
Alessia Claps • Martina della Corte • \\ Simona Gerocarni Nappo • Paola Francalanci • \\ Paolo Palma • Andrea Finocchi
}

Received: 14 March 2014 /Revised: 30 May 2014 / Accepted: 6 June 2014 /Published online: 19 July 2014

(C) IPNA 2014

\begin{abstract}
Background Chronic granulomatous disease (CGD) is a primary immunodeficiency resulting from the absence or malfunction of oxidative mechanism in phagocytic cells. The disease is due to a mutation in one of four genes that encode subunits of the nicotinamide adenine dinucleotide phosphate (NADPH) oxidase complex. Affected patients experience severe infections and granuloma formation due to exuberant inflammatory responses. Some evidence suggests that eosinophilic cystitis (EC) is included in the spectrum of inflammatory manifestations. EC is an inflammatory disease, rare in childhood, which may require different, nonstandardized therapeutic approaches, ranging from antihistamines to cyclosporine.
\end{abstract}

Paolo Palma and Andrea Finocchi shared last authorship

Electronic supplementary material The online version of this article (doi:10.1007/s00467-014-2883-7) contains supplementary material, which is available to authorized users.

A. Claps $\cdot$ M. della Corte $\cdot$ P. Palma $\cdot$ A. Finocchi

Unit of Immunology and Infectious Diseases, University-Hospital

Pediatric Department, Bambino Gesù Children Hospital, IRCCS,

Rome, Italy

S. Gerocarni Nappo

Department of Nephrology and Urology, Bambino Gesù Children

Hospital, IRCCS, Rome, Italy

P. Francalanci

Department of Pathology, Bambino Gesù Children Hospital, IRCCS,

Rome, Italy

A. Finocchi $(\bowtie)$

Unit of Immunology and Infectious Diseases, University Hospital Department of Pediatrics, Bambino Gesù Children's Hospital and University of Rome "Tor Vergata", Piazza Sant'Onofrio,

400165 Rome, Italy

e-mail: andrea.finocchi@uniroma2.it
Case-Diagnosis/Treatment Herein we describe the cases of two CGD patients with CGD who experienced EC during hospitalization for a severe infection.

Conclusions EC in immunocompetent children seems to have a self-limiting course, unlike in CGD patients, in whom it presents a prolonged and recurrent course. We focus on the effective therapy administered to our patients with CGD and review the corresponding literature.

\section{Introduction}

Chronic granulomatous disease (CGD) is an uncommon immunodeficiency disorder of phagocyte oxidative metabolism. To date, the cases of only six CGD patients suffering from eosinophilic cystitis (EC) have been described in the literature. We report our experience with two additional cases of EC in CGD patients who presented irritative urinary symptoms during life-threatening infections.

\section{Case reports}

Patient 1

A 3-and-a-half-year-old boy was transferred to our hospital with a diagnosis of multiple brain abscesses [1]. His past medical history was significant for recurrent episodes of enteritis caused by Salmonella typimurium. Immunological analysis, including nitro-blu tetrazolium (NBT) test and dihydrorhodamine 1,2,3 assay (DHR), revealed the reduction of oxidase granulocyte activity. Mutation of the $C Y B B$ gene confirmed the diagnosis of X-linked CGD. Combined antibiotic therapy was started, resulting in a progressive improvement of radiological and neurological pictures. However, on the 18th day of hospitalization, the child developed dysuria, 
suprapubic pain, dribbling, urinary urgency and incontinence. The urological evaluation revealed penile phimosis and suprapubic tenderness. He was afebrile. Urinalysis showed albuminuria and microscopic hematuria with no evidence of pyuria. Urine culture was negative, as were the bacterioscopic evaluation and polymerase chain reaction (PCR) for Mycobacterium spp. Laboratory examination revealed white blood cell (WBC) count of $10.140 / \mathrm{mm}^{3}$, with $61.4 \%$ neutrophils, $2.4 \%$ eosinophils, $27.2 \%$ lymphocytes, and $6.7 \%$ monocytes. Neither eosinophilia nor eosinophiluria was detected. Inflammatory markers were elevated, with a C-reactive protein (CRP) level of $7.65 \mathrm{mg} / \mathrm{dl}$ (normal value $0-0.5 \mathrm{mg} / \mathrm{dl}$ ) and erythrocyte sedimentation rate (ESR) of 16 (normal value $0-15 \mathrm{~mm} / \mathrm{h}$ ). Kidney-function tests were normal. Renal sonography revealed normal kidneys in terms of size, shape, and echogenicity, and there was no evidence of urinary obstruction or hydronephrosis. However, bladder sonography displayed an irregular and marked thickening of the wall, with evidence of hyperemia on color-Doppler examination (Electronic Supplementary Fig. 1a). Therefore, a cystoscopic examination and bladder biopsy were performed.

Histological analysis of biopsy specimens demonstrated erosion of the epithelium lining, with aspects of reactive hyperplasia and chronic inflammation with eosinophilic infiltrate. No granulomas were observed in the specimens. Cultures obtained from bladder biopsy were sterile. During the cystoscopic procedure, intravesical instillation of dexamethasone was performed and a bladder catheter placed. Treatment was instituted with prolonged administration of cetirizine ( $5 \mathrm{mg}$ per day) associated with intravesical instillation of dexamethasone (16 mg per day) for 7 days. His symptoms disappeared, and repeated sonography over the next 2 weeks showed complete resolution of bladder-wall thickening (Electronic Supplementary Fig. 1b). The patient remains recurrence-free 1 year after diagnosis.

\section{Patient 2}

We present the case of a 3-year-old boy referred to our clinic because of fever and pre-existing lung disease who had been diagnosed with CGD at the age of 2 years (diagnosis based on NBT test and genetic confirmation of $C Y B B$ gene mutation) when he was examined on account of his history of recurrent pneumonia, lymphadenitis and poor growth. We performed a chest computed tomography (CT) scan, which showed no change compared with the previous one, and a CT-guided lung biopsy showed a histological picture of interstitial pneumonia and pleurisy. Microbiological and PCR investigations were negative. An empirically based antibiotic and antimycotic therapy was started. Because of the persistent clinical and radiological picture, after 1 month of hospitalization, the patient successfully underwent a left lower lobectomy. After 10 days of clinical improvement, the child developed micturition pain, frequency, and urinary incontinence, with no evidence of urinary infection. Urine culture was negative. Laboratory examination revealed WBC count of 5.520/mm3, with $42.8 \%$ neutrophils, $4.8 \%$ eosinophils, $34.4 \%$ lymphocytes, $12.9 \%$ monocytes, CRP $5.79 \mathrm{mg} / \mathrm{dl}$ (normal value $0-0.5 \mathrm{mg} / \mathrm{dl}$ ), and ESR $100 \mathrm{~mm} / \mathrm{h}$ (normal value $2-20 \mathrm{~mm} / \mathrm{h}$ ); renal-function indices were normal. Renal sonography revealed normal kidneys in terms of size, morphology, and echogenicity, and no evidence of urinary obstruction or urinary tract malformations. However, bladder sonography demonstrated the presence of an echogenic and nonmobile lesion, adherent to the bladder neck, initially interpreted as a blood clot. Therefore, cystoscopic examination and bladder biopsy were performed. Cystoscopic examination showed the presence of a chronic inflammation of the bladder and the biopsy indicated the presence of an eosinophilic infiltrate, with no evidence of hyphae or fungal spores on periodic acid-Schiff(PAS) stain (Electronic Supplementary Fig. 1c). No granulomas were observed in the specimen. Treatment with intravesical instillation of dexamethasone (16 mg per day for 7 days) and prolonged administration of cetirizine ( $5 \mathrm{mg}$ per day) was instituted. After 7 days, bladder sonography showed lesion healing. The patient remains symptom free after 7 years' follow-up.

\section{Discussion and review of literature}

CGD is an uncommon primary immunodeficiency of Xlinked or autosomal inheritance arising from a defect of "respiratory burst" in phagocytic cells. Affected patients are susceptible to severe life-threatening bacterial and fungal infections and exuberant inflammation, leading to obstructive granuloma. The mechanism underlying the abnormal inflammatory response in CGD is poorly defined. Sometimes, granulomatous complication derives from an unresolved infection with the persistence of antigen within the granuloma. However, as the inflammatory sites are usually sterile, the pathogenetic mechanism may relate to delayed apoptosis of CGD neutrophils, with diminished production of antiinflammatory mediators. Further underlying mechanisms implicated are: unbalanced innate immune receptors, altered Tcell-surface redox levels, induction of Th17 cells, decreased autophagy, and increased inflammasome activation [2]. In the genitourinary tract, the most common manifestation is granulomatous cystitis, which may be complicated by ureteral obstruction and, consequently, by hydroureteronephrosis [3]. Some reports, including our experience, demonstrate that genitourinary involvement in CGD may also be represented by EC.

$\mathrm{EC}$ is a rare pediatric inflammatory disorder of the bladder characterized by extensive eosinophilic infiltration of all 


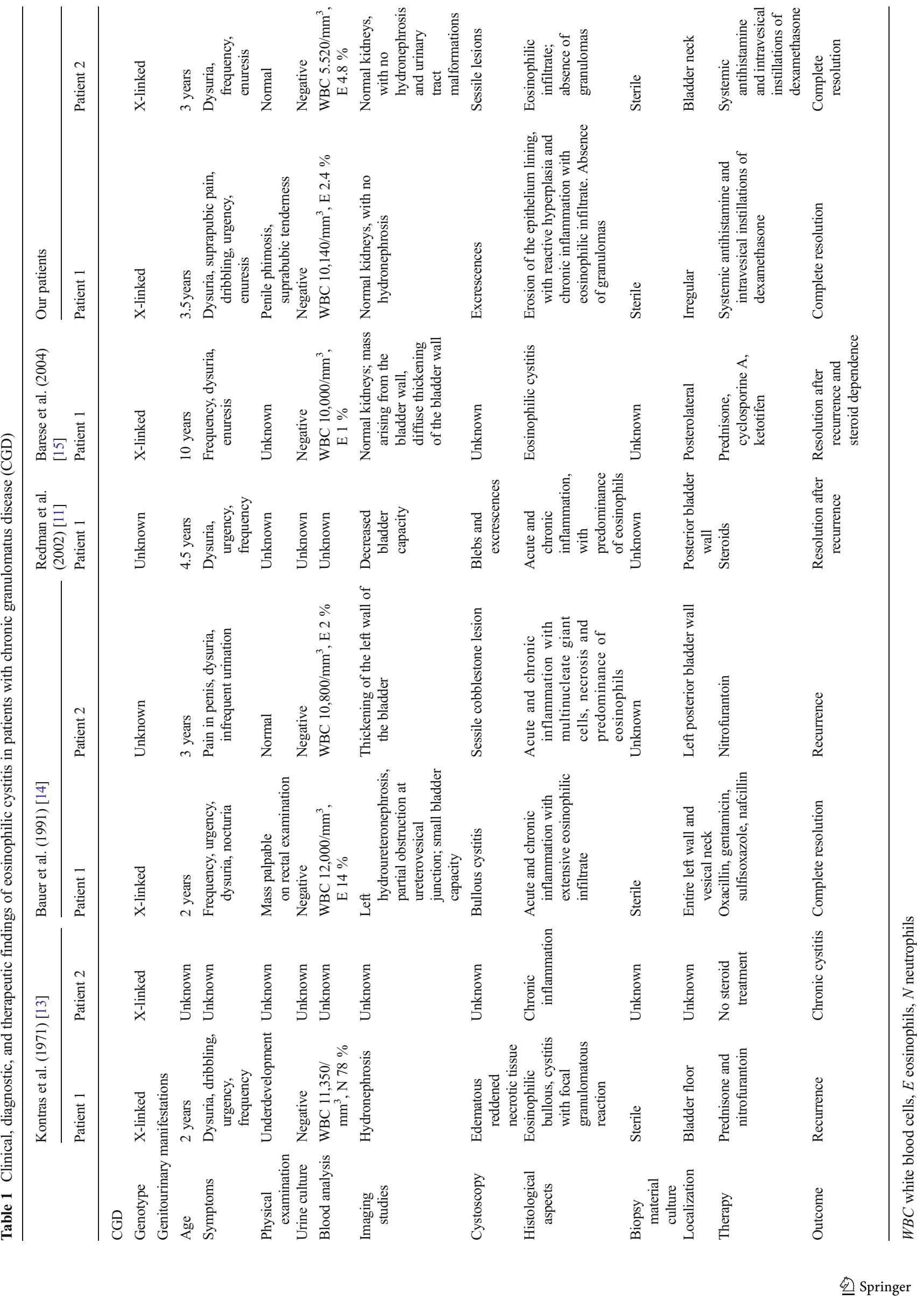


layers of the bladder wall [4]. EC seems to be related to dysregulation of the immune system, as suggested by reports of EC occurring in patients with a history of allergies [5-8] or subsequent to drug administration (penicillin, mitomycin, clometacin, cyclophosphamide, coumadin, tranilast, and thiotepa) $[5,6]$. The detection of transmural eosinophilic infiltration should also prompt suspicion of a differential diagnosis, including parasitosis, tumors, tuberculous cystitis, and bladder instrumentation $[4,9]$. Is not clear whether the EC in CGD patients depends on the natural course of the immunodeficiency related to hyperinflammation or if it develops in response to other triggers, such as drugs chronically used in these patients.

EC usually presents with symptoms of irritative nature, such as dysuria, hematuria, urgency, frequency, and suprapubic tenderness $[4,5,7,9]$. Patients may also present with an obstructive bladder mass resembling malignancy and causing hydroureteronephrosis $[5,10]$. Optimal treatment of EC is not standardized. Multiple treatment modalities have been tried with variable success. Various combinations of antibiotics, antihistamines, nonsteroidal anti-inflammatory molecules, and steroids are reported to be effective. However, spontaneous remission has been frequently seen in children, suggesting observation only $[5-7,9,11,12]$. We carried out a retrospective literature review of reported CGD patients who developed $\mathrm{EC}$ in order to assess clinical manifestations, diagnostic tools, therapeutic strategies and outcomes (Table 1) [11, 13-15].

In this series, all the patients were boys and were mainly affected by the X-linked variant of CGD. The onset of urinary manifestations generally occurred in early childhood, except for the patient described by Barese et al. [15], who was 10 years old. Mean patient age was 48 (median 36; range 24 120) months. Presenting symptoms were typical and were represented in all patients by dysuria, increased frequency or urgency and enuresis, variably accompanied by urinary dribbling, penile pain, infrequent urination and suprapubic pain. Peripheral blood eosinophilia and eosinophiluria were not present in the majority of cases, and microbiological analyses performed on urine and bladder specimens were negative. Diagnosis of EC was made in all CGD patients only after cystoscopy and histological examinations, which confirmed inflammation of the bladder wall with a prevalent eosinophilic infiltrate. Interestingly, in all reported patients, cystoscopy revealed the presence of sessile lesions that affected, with a slight predominance, the posterior bladder wall. Unlike immunocompetent children in whom EC has a benign course, CGD patients frequently experience recurrence, requiring different treatments, including antibiotics, steroids, and antihistamines. Only in the first case described by Bauer et al. [14] a combined antibiotic therapy ensured complete disease resolution; the other patients, who were treated exclusively with antimicrobials, experienced recurrence of the clinical picture. Steroids were effective in all other cases, except for the patient reported by Barese et al. [15], who developed a steroid dependence and recovered only after antihistamine administration.

Prolonged and recurrent course of EC in children should lead to evaluation of CGD diagnosis. This implies targeted therapeutic interventions that will not further undermine the already compromised immune system, such as high-dose systemic corticosteroids. In our experience, patients were successfully treated with antihistamines orally and a short course of steroids via intravesical instillation. This approach ensured a rapid resolution of inflammatory lesions, as demonstrated by ultrasound images after 1 week of therapy, and did not lead to a worsening of the underlying infectious picture characterized in the first case by multiple cerebral abscesses and in the second by severe pneumonia. Furthermore, no systemic impact on the adrenocorticotropic hormone (ACTH) pathway was observed. Thus, based on our experience, we suggest that for such patients with EC, intravesical instillation of corticosteroids coupled with oral administration of antihistamine should be considered as the first approach to induce remission and prevent recurrence of the disorder.

Disclosures None.

\section{References}

1. Finocchi A, Claps A, Serafinelli J, Salfa I, Longo D, Di Matteo G, Aiuti A, Rossi P (2014) Chronic granulomatous disease presenting with salmonella brain abscesses. Pediatr Infect Dis J 33:525-528

2. Rieber N, Hector A, Kuijpers T, Roos D, Hartl D (2012) Current concepts of hyperinflammation in chronic granulomatous disease. Clin Dev Immunol 2012:252460

3. Holland SM (2010) Chronic granulomatous disease. Clin Rev Allergy Immunol 38:3-10

4. Pomeranz A, Eliakim A, Uziel Y, Gottesman G, Rathaus V, Zehavi T, Wolach B (2001) Eosinophilic cystitis in a 4-year-old boy: successful long-term treatment with cyclosporin A. Pediatrics 108:e113

5. Thompson RH, Dicks D, Kramer SA (2005) Clinical manifestations and functional outcomes in children with eosinophilic cystitis. J Urol 174:2347-2349

6. Verhagen PC, Nikkels PGJ, de Jong TPVM (2001) Eosinophilic cystitis. Arch Dis Child 84:344-346

7. Sparks S, Kaplan A, DeCambre M, Kaplan G, Holmes N (2013) Eosinophilic cystitis in the pediatric population: a case series and review of the literature. J Pediatr Urol 9:738-744

8. Sano K, Terashima K, Gotoh K, Ijiri R, Tanaka Y (2000) A case of eosinophilic cystitis in a 5-year-old boy. Int J Urol 7:54-57

9. Grønlund A, Glenthøj A, Kvist E (1999) Eosinophilic cystitis- diagnosis and treatment in Denmark. Scand J Urol Nephrol 33:321-323

10. Thomas JC, Ross JH (2004) Eosinophilic cystitis in a child presenting with a bladder mass. J Urol 171:1654-1655

11. Redman JF, Parham DM (2002) Extensive inflammatory eosinophilic bladder tumors in children: experience with three cases. South Med J 95:1050-1052 
12. Collman RJ, Dickeerman JD (1990) Corticosteroids in the management of cystitis secondary to chronic granulomatous disease. Pediatrics 85:219-221

13. Kontras SB, Bodenbender JG, McClave CR, Smith JP (1971) Interstitial cystitis in chronic granulomatous disease. J Urol 105: $575-578$
14. Bauer SB, Kogan SJ (1991) Vesical manifestations of chronic granulomatous disease in children. Its relation to eosinophilic cystitis. Urology 37:463-466

15. Barese CN, Podestá M, Litvak E, Villa M, Rivas EM (2004) Recurrent eosinophilic cystitis in a child with chronic granulomatous disease. J Pediatr Hematol Oncol 26:209-212 